\title{
Fragilisation par le zinc liquide des aciers haute résistance pour l'automobile
}

\section{Liquid zinc embrittlement of high strength automotive steels}

\author{
Renaud Frappier ${ }^{1,2}$, Pascal Paillard ${ }^{1}$, René Le Gall ${ }^{1}$, Thomas Dupuy ${ }^{2}$, Damien Fabregue ${ }^{3}$ \\ et Xavier Kleber ${ }^{3}$
}

\author{
${ }^{1}$ Institut des Matériaux Jean Rouxel UMR6502, Site de la Chantrerie, BP. 50609, 44306 Nantes, France \\ 2 ArcelorMittal R\&D, Automotive Products, Voie Romaine, BP. 30320, 57283 Maizières-lès-Metz, France \\ 3 INSA-Lyon, MATEIS UMR5510, 69621 Villeurbanne, France
}

\begin{abstract}
This study deals with liquid zinc embrittlement for electro-galvanized steel. Mechanical characterization by high temperature tensile tests shows a drastic loss of ductility between $700{ }^{\circ} \mathrm{C}$ and $950^{\circ} \mathrm{C}$. SEM investigations show that steel grain boundaries under the steel/coating interface are penetrated by a liquid $\mathrm{Zn}$ channel, only in the temperature range of embrittlement. A correlation can be drawn between i) thermal activated-grain boundary wetting and ii) crack propagation in presence of external stress.

Résumé. Cette étude présente les investigations menées sur la fragilisation par le zinc liquide d'un acier électro-zingué. La caractérisation mécanique par essais de traction à haute température montre un important puits de ductilité entre environ $700^{\circ} \mathrm{C}$ et environ $950^{\circ} \mathrm{C}$. L'observation au MEB des éprouvettes de traction indique que, dans la gamme de température observée pour laquelle il y a fragilisation, on a mouillage intergranulaire des joints de grains de l'acier à l'interface acier/revêtement par des films de $\mathrm{Zn}$. La corrélation entre mouillage intergranulaire thermiquement activé d'une part, et propagation de fissure lors du chargement d'autre part, est discutée.
\end{abstract}

\section{INTRODUCTION}

Les aciers à haute résistance (HR) pour l'automobile ont été développés depuis les vingt dernières années dans le cadre de l'allégement des structures, problématique encore actuelle de l'industrie des matériaux pour le transport. La maitrise des compositions chimiques et des traitements thermomécaniques permettent aujourd'hui aux aciéristes d'obtenir des microstructures multiphasées, par exemples dual phase (DP) ou transformation inducedplasticity (TRIP), nécessaires au compromis entre haute résistance, formabilité, et coût (aciers faiblement alliés). La galvanisation, à chaud ou par voie électrolytique, consiste à revêtir la tôle d'acier d'une couche de $\mathrm{Zn}$ et permet la résistance à la corrosion des produits automobiles.

Plusieurs études récentes ont montré que certains aciers galvanisés peuvent présenter des fissures de surface à l'issue des étapes d'emboutissage à chaud ou de soudage, dans des gammes de température où le revêtement devient liquide. Etant donné que, dans les mêmes conditions, les produits non revêtus ne sont pas sujet à cette fissuration, le phénomène de fragilisation par les métaux liquides (FML) a été mis en avant comme cause de l'apparition des défauts.

La FML peut être définie comme la perte de ductilité d'un métal solide lorsqu'il est déformé en contact avec un autre métal à l'état liquide. Ce phénomène est toujours source de nombreux débats scientifiques. En effet, plusieurs critères empiriques et mécanismes théoriques ont été proposés depuis 50 ans mais ils sont souvent contradictoires et ne couvrent pas l'ensemble des observations expérimentales. Les études sur la fragilisation des aciers ou du fer par le zinc sont peu nombreuses et ne permettent pas une résolution du problème industriel. On peut néanmoins établir que la FML se manifeste sous déformation plastique par la propagation de fissure intergranulaire causée par la présence de zinc en fond de fissure.

Aujourd'hui la FML peut être empêchée par une bonne maitrise des procédés de mises en forme et d'assemblage des aciers. Néanmoins la compréhension des phénomènes est nécessaire pour le développement serein des futures nuances d'aciers. On se propose ici d'étudier le comportement mécanique à haute température d'un acier galvanisé par essai de traction à haute vitesse de déformation pour reproduire les conditions subies par le matériau lors des processus de mise en œuvre. On fera ensuite le lien entre la caractérisation mécanique et les observations microstructurales réalisées.

\section{MATÉRIAU ET MÉTHODES EXPÉRIMENTALES}

On étudie un acier de $1 \mathrm{~mm}$ d'épaisseur revêtu d'une couche quasi-pure de $\mathrm{Zn}$ d'environ $12 \mu \mathrm{m}$ d'épaisseur déposé par voie électrolytique. La microstructure, à température ambiante, est composée d'une matrice ferritique et bainitique avec environ $20 \%$ d'austénite résiduelle. La composition chimique indicative est : 0,2 C - 1,6 Mn - 1,6 Si.

Les éprouvettes de traction de longueur utile $35 \mathrm{~mm}$ et de largeur $8 \mathrm{~mm}$ sont placées dans une machine d'essai 


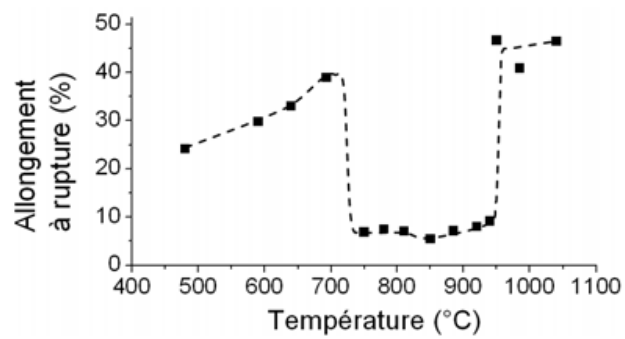

Figure 1. Trou de ductilité entre $700^{\circ} \mathrm{C}$ et $950^{\circ} \mathrm{C}$.

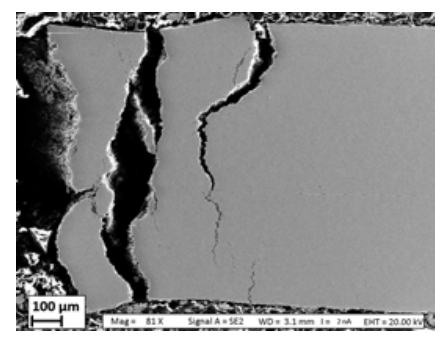

Figure 2. Eprouvette rompue à $880^{\circ} \mathrm{C}$.

thermomécanique Gleeble ${ }^{\circledR}$. L'éprouvette est chauffée pendant une seconde pour atteindre la température de l'essai et déformée immédiatement à la vitesse de $1,210^{-1} \mathrm{~s}^{-1}$ puis trempée à l'eau après $5 \mathrm{~s}$ à haute température. On réalise les essais à différentes températures entre $400^{\circ} \mathrm{C}$ et $1100^{\circ} \mathrm{C}$. La température est régulée à l'aide d'un thermocouple de type $\mathrm{K}$ soudé au milieu de l'éprouvette. Contraintes et déformations sont calculées à partir du déplacement de la traverse et du capteur de force.

Les observations microstructurales sont faites sur des coupes polies longitudinales des éprouvettes de traction dans un microscope électronique à balayage à canon à effet de champ Merlin de la société Zeiss.

\section{RÉSULTATS ET DISCUSSION}

\section{Perte de ductilité en traction}

La figure 1 présente l'élongation maximale des éprouvettes de traction en fonction de la température. On peut voir une perte de ductilité très importante entre $700^{\circ} \mathrm{C}$ et $950^{\circ} \mathrm{C}$. La ductilité résiduelle est alors d'environ $5 \%$. La figure 2 présente une partie de la coupe longitudinale d'une demiéprouvette après rupture. En plus de la fissure traversante ayant causé la rupture (à gauche de l'image), on peut y voir plusieurs fissures secondaires très profondes.

\section{MOUILLAGE INTERGRANULAIRE}

L'observation de l'interface entre l'acier et le revêtement hors des zones de fissuration montre une morphologie de type mouillage intergranulaire comme représentée sur la figure 3. La figure 4 présente l'évolution de la profondeur de mouillage apparente en fonction de la température.

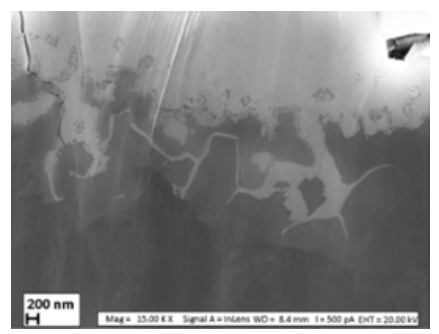

Figure 3. Interface acier/revêtement après $5 \mathrm{~s}$ à $880^{\circ} \mathrm{C}$. Mouillage intergranulaire par le $\mathrm{Zn}$.

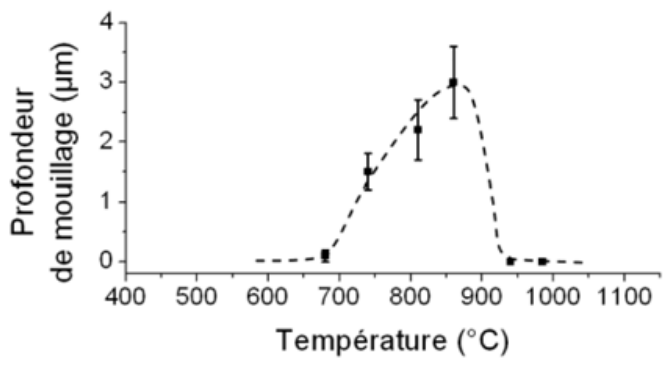

Figure 4. Influence de la température sur le mouillage intergranulaire.

\section{LIEN ENTRE MOUILLAGE ET PROPAGATION DE FISSURE}

Le mouillage intergranulaire, phénomène thermiquement activé, peut être décrit simplement en considérant l'énergie interfaciale solide/liquide $\gamma_{s l}(\mathrm{~T})$ et l'énergie du joint de grains $\gamma_{j d g}(\mathrm{~T})$. Une transition de mouillage apparait pour :

$$
2 \gamma_{\mathrm{sl}}(\mathrm{T})=\gamma_{\mathrm{jdg}}(\mathrm{T})
$$

Le remplacement du joint de grain par un film liquide, i.e. le mouillage parfait, est alors énergétiquement favorable. Les résultats obtenus indiquent qu'une transition de mouillage se produit pour notre système acier/zinc autour de $700^{\circ} \mathrm{C}$. On a aussi établit le lien entre le mouillage observé et la gamme de température pour la fragilisation.

L'application d'une déformation macroscopique se traduit par des contraintes locales importantes au fond du sillon de mouillage. La cinétique du phénomène de mouillage, avec une vitesse de pénétration de l'ordre de $1 \mu \mathrm{m} \mathrm{s}^{-1}$ dans le cas sans contrainte extérieure, n'est plus retrouvée. La propagation d'une fissure est alors guidée par un mécanisme très local de fragilisation du joint de grain en fond de fissure. Ce mécanisme conduit à des cinétiques de propagation de fissure de l'ordre de $1 \mathrm{~mm} \mathrm{~s}^{-1}$ et donc de plusieurs ordres de grandeur supérieurs aux cinétiques de pénétration par mouillage.

Deux points importants sont donc à considérer pour mieux comprendre le phénomène de fragilisation par les métaux liquides :

i) Le mouillage intergranulaire, situation d'équilibre mécanique macroscopique, et la propagation de fissure, situation hors équilibre mécanique, doivent être distingués. 
ii) Etant donné que la gamme de température permettant la propagation de fissure est conditionnée par le mouillage intergranulaire, le mécanisme local de propagation de fissure doit être basé sur les mêmes mécanismes fondamentaux que le mouillage. Adsorption ? Diffusion intergranulaire ? Dissolution préférentielle du joint de grain dans le liquide?

\section{CONCLUSION}

Cette étude a permis de caractériser la perte de ductilité en traction d'un acier galvanisé causée par la FML. Le lien entre fragilisation, mouillage intergranulaire et propagation de fissure a été établi.

Cette étude peut constituer la base pour la définition de contre-mesures métallurgiques permettant de diminuer la sensibilité des aciers à la FML et de résoudre les problèmes apparaissant dans le process de fabrication et de mise en œuvre des aciers.

\section{Références}

H Kamdar, Embrittlement by liquid metals, School of engineering and applied science, University of California, Los Angeles, 1972.

S.P. Lynch, Failure of structures and components by metal-induced embrittlement, Journal of Failure Analysis and Prevention, 2008, 8, 259-274.

C. Beal, et al., Embrittlement of a zinc coated high manganese TWIP steel, Materials Science and Engineering A, 2012, 543, 76-83.

C.W. Lee, Liquid-metal-induced embrittlement of Zncoated hot stamping steel, Metallurgical and Materials Transactions A, 2012, 43, 5122-5127

E.E. Glickman, Grain boundary grooving accelerated by local plasticity as a possible mechanism of liquid metal embrittlement, Interface Science, 2003, 11, 451-459

K. Wolski, V. Laporte, Grain boundary diffusion and wetting in the analysis of intergranular penetration, Materials Science and Engineering A, 2008, 495, 138146 